\title{
The Gerber-Shiu Discounted Penalty Function in the Stationary Renewal Risk Model
}

by

\author{
Gordon E Willmot \\ University of Waterloo \\ and \\ David C M Dickson \\ The University of Melbourne \\ RESEARCH PAPER NUMBER 102 \\ August 2002
}

Centre for Actuarial Studies

Department of Economics

The University of Melbourne

Victoria 3010

Australia 


\title{
The Gerber-Shiu discounted penalty function in the stationary renewal risk model
}

\author{
Gordon E. Willmot and David C.M. Dickson
}

\begin{abstract}
The discounted penalty function introduced by Gerber and Shiu (1998) is considered in the stationary renewal risk model, where it is expressed in terms of the same discounted penalty function in the ordinary renewal risk model. This relationship unifies and generalizes known special cases. An invariance property between the stationary renewal risk model and the classical Poisson model with respect to the ruin probability is also generalized as a result.
\end{abstract}

Keywords Sparre Andersen model, severity of ruin, deficit at ruin, Laplace transform, Lundberg's fundamental equation

Acknowledgments Support for the first author from the Munich Reinsurance Company is gratefully acknowledged, as was support by a grant from the Natural Sciences and Engineering Research Council of Canada.

\section{Introduction and background}

Various properties associated with the stationary renewal risk or stationary Sparre Andersen process are examined in this paper.

We begin by introducing the (ordinary) renewal risk model. The number of claims process $\left\{N_{t} ; t \geq 0\right\}$ is assumed to be a renewal process where the independent and identically distributed (iid) sequence $\left\{V_{1}, V_{2}, \ldots\right\}$ of absolutely continuous positive random variables represents the interclaim times. That is, $V_{1}$ is the time until the first claim occurs, and $V_{i}$ is the time between the $(i-1)$-th and the $i$-th claim for $i=2,3,4, \ldots$ Let $V_{1}$ have distribution function (df) $K(t)=1-\bar{K}(t)=\operatorname{Pr}(V \leq t)$ where $V$ is an arbitrary $V_{i}$. Let $k(t)=K^{\prime}(t)$ be the associated probability density function (pdf) and $E(V)=\int_{0}^{\infty} \bar{K}(t) d t<\infty$. 
The individual claim amounts $\left\{Y_{1}, Y_{2}, \ldots\right\}$ are assumed to be iid positive random variables with df $H(y)=1-\bar{H}(y)=\operatorname{Pr}(Y \leq y)$, with $Y$ an arbitrary $Y_{i}$, itself representing the $i$-th claim. Let $E(Y)=\int_{0}^{\infty} \bar{H}(y) d y<E(V)$, and $\tilde{h}(s)=E\left(e^{-s Y}\right)=\int_{0}^{\infty} e^{-s y} d H(y)$. It is convenient to introduce the equilibrium or integrated tail df as $H_{1}(y)=1-\bar{H}_{1}(y)=\int_{0}^{y} \bar{H}(t) d t / E(Y)$, and $\tilde{h}_{1}(s)=\int_{0}^{\infty} e^{-s y} d H_{1}(y)=\{1-\tilde{h}(s)\} /\{s E(Y)\}$.

Premiums are paid continuously at rate $c=(1+\theta) E(Y) / E(V)$ per unit time, where $\theta>0$ in the relative security loading. The insurer's surplus at time $t$ is defined as $\left\{U_{t} ; t \geq 0\right\}$, where $U_{t}=u+c t-\sum_{i=1}^{N_{t}} Y_{i}$, and $u \geq 0$ is the initial surplus. The time of ruin is $T=\inf \left\{t: U_{t}<0\right\}$ where $T=\infty$ if $U_{t} \geq 0$ for all $t \geq 0$. If ruin occurs, the deficit at ruin is $\left|U_{T}\right|$ and the surplus immediately before ruin is $U_{T-}$. The probability of ruin is $\psi(u)=$ $\operatorname{Pr}(T<\infty)=E\{I(T<\infty)\}$ where $I(A)=1$ if $A$ occurs and $I(A)=0$ otherwise. See Grandell (1991, Section 3.1), Embrechts et al (1997, Chapter 1), Rolski et al (1999, Section 6.5), Asmussen (2000, Chapter V), Willmot and Lin (2001, Chapter 11), and references therein for details on this well known model.

The stationary or equilibrium renewal process is identical in all respects to the (ordinary) renewal risk model except that the time until the first claim occurs has pdf $\bar{K}(t) / E(V)$ rather than $k(t)$. For this process, let $T_{e}$ denote the time of ruin, and $\psi_{e}(u)=\operatorname{Pr}\left(T_{e}<\infty\right)=E\left\{I\left(T_{e}<\infty\right)\right\}$ the ruin probability. It is well known that

$$
\psi_{e}(u)=\frac{1}{1+\theta} \int_{0}^{u} \psi(u-t) d H_{1}(t)+\frac{1}{1+\theta} \bar{H}_{1}(u), \quad u \geq 0 .
$$

See Grandell (1991, pp. 67-9) or Willmot and Lin (2001, p. 231), for example. If ruin occurs, let $\left|U_{T_{e}}\right|$ and $U_{T_{e}-}$ denote the deficit at ruin and the surplus immediately before ruin, respectively. In this paper we shall generalize (1.1) by replacing the expectation of the indicator function by that of the Gerber-Shiu discounted penalty function, to be introduced below.

The classical Poisson model is a special case of each of the above two models obtained with $K(t)=1-e^{-\lambda t}$, and $E(V)=1 / \lambda$. In this case we let $T_{*}$ denote the time of ruin, $\psi_{*}(u)=\operatorname{Pr}\left(T_{*}<\infty\right)=E\left\{I\left(T_{*}<\infty\right)\right\}$ the ruin probability, and if ruin occurs $\left|U_{T_{*}}\right|$ and $U_{T_{*}-}$ the deficit at ruin and the surplus immediately before ruin, respectively. It is well known that

$$
\psi_{e}(0)=\psi_{*}(0)=\frac{1}{1+\theta}
$$

an invariance property between the stationary renewal risk and classical models. This result will also be generalized via the Gerber-Shiu discounted penalty function, which is now discussed. 
Let $\delta \geq 0$ be a discount factor, and $w\left(x_{1}, x_{2}\right), x_{1} \geq 0, x_{2} \geq 0$, a nonnegative function. Then Gerber and Shiu (1998) introduced the so-called Gerber-Shiu discounted penalty function

$$
m_{*}(u)=E\left\{e^{-\delta T_{*}} w\left(U_{T_{*}-},\left|U_{T_{*}}\right|\right) I\left(T_{*}<\infty\right)\right\},
$$

viewed as a function of the initial surplus $u$. The terminology is unnecessarily restrictive, since $\delta$ may also be viewed as a Laplace transform argument, and $w$ a convenient function of the surplus and deficit at ruin, rather than simply a penalty at ruin. See Lin and Willmot $(1999,2000)$, for example. Many properties of the surplus process may be obtained from this very general function $m_{*}(u)$, and $m_{*}(u)$ reduces to $\psi_{*}(u)$ if $\delta=0$ and $w\left(x_{1}, x_{2}\right)=1$.

Let $\rho=\rho(\delta)$ be the unique nonnegative solution of the equation

$$
c \rho+\lambda \tilde{h}(\rho)=\lambda+\delta,
$$

where $\rho(0)=0$. The Gerber and Shiu (1998) demonstrated that $m_{*}(u)$ satisfies a defective renewal equation. That is, let

$$
\phi=\frac{\tilde{h}_{1}(\rho)}{1+\theta},
$$

so that $0<\phi<1$, and the df $B(x)=1-\bar{B}(x)$ be defined by

$$
\bar{B}(x)=\frac{\int_{0}^{\infty} e^{-\rho t} \bar{H}(x+t) d t}{\int_{0}^{\infty} e^{-\rho t} \bar{H}(t) d t}, \quad x \geq 0
$$

Then

$$
m_{*}(u)=\phi \int_{0}^{u} m_{*}(u-t) d B(t)+\alpha(u), \quad u \geq 0,
$$

where

$$
\alpha(u)=\frac{e^{\rho u}}{(1+\theta) E(Y)} \int_{u}^{\infty} e^{-\rho t} \int_{t}^{\infty} w(t, y-t) d H(y) d t .
$$

See also Lin and Willmot (1999).

The Gerber-Shiu discounted penalty function in the (ordinary) renewal risk model is defined by

$$
m(u)=E\left\{e^{-\delta T} w\left(U_{T-},\left|U_{T}\right|\right) I(T<\infty)\right\},
$$

and this may be obtained for certain choices of $K(t)$ and/or $w\left(x_{1}, x_{2}\right)$. For example, Dickson and Hipp (2001) demonstrate how $m(u)$ may be evaluated if $K(t)$ is from the Coxian-2 or phase type-2 class when $w\left(x_{1}, x_{2}\right)=1$. The case with $\delta=0$ and $w\left(x_{1}, x_{2}\right)=x_{2}^{k}$ is discussed by Drekic et al (2002). 
For the stationary renewal risk model, (1.9) is replaced by

$$
m_{e}(u)=E\left\{e^{-\delta T_{e}} w\left(U_{T_{e}-},\left|U_{T_{e}}\right|\right) I\left(T_{e}<\infty\right)\right\} .
$$

In the next section we express $m_{e}(u)$ in terms of $m(u)$, an expression which reduces to (1.1) as a special case. Other useful relationships between the deficit at ruin and surplus prior to ruin are also obtained, and these are examined in Section 3, where it is shown that considerable simplification occurs when $\delta=0$.

Finally, the original Gerber-Shiu result is recovered easily in Section 4, and the invariance relation (1.2) is substantially generalized.

\section{The general relationship}

The following property of Laplace transforms will be used repeatedly in what follows.

Proposition 2.1 If $\tilde{a}(s)=\int_{0}^{\infty} e^{-s u} a(u) d u$, then for $r \neq s$,

$$
\frac{\tilde{a}(r)-\tilde{a}(s)}{s-r}=\int_{0}^{\infty} e^{-s u}\left\{e^{r u} \int_{u}^{\infty} e^{-r t} a(t) d t\right\} d u .
$$

Proof: Straightforward by integration by parts on the right side of (2.1).

Next, we condition on the time and amount of the first claim to obtain an integral equation satisfied my $m(u)$ in the (ordinary) renewal risk case, namely

$$
m(u)=\int_{0}^{\infty} e^{-\delta t} \gamma(u+c t) k(t) d t
$$

where

$$
\gamma(t)=\int_{0}^{t} m(t-y) d H(y)+\int_{t}^{\infty} w(t, y-t) d H(y) .
$$

A change in the variable of integration in (2.2) results in

$$
m(u)=\frac{1}{c} \int_{u}^{\infty} e^{-\delta\left(\frac{t-u}{c}\right)} k\left(\frac{t-u}{c}\right) \gamma(t) d t .
$$

The integral equation (2.4) may be used to solve for $m(u)$ for some choices of $k(\cdot)$ using the approach of Dickson and Hipp (2001).

For the stationary process, $(2.2)$ is replaced by

$$
m_{e}(u)=\int_{0}^{\infty} e^{-\delta t} \gamma(u+c t) \frac{\bar{K}(t)}{E(V)} d t
$$


since the stationary renewal risk process behaves like the (ordinary) renewal risk process upon the occurrence of the first claim. A similar change of variable in (2.5) implies that

$$
m_{e}(u)=\frac{1}{c E(V)} \int_{u}^{\infty} e^{-\delta\left(\frac{t-u}{c}\right)} \bar{K}\left(\frac{t-u}{c}\right) \gamma(t) d t .
$$

By noting that

$$
\frac{d}{d u}\left\{e^{-\delta\left(\frac{t-u}{c}\right)} \bar{K}\left(\frac{t-u}{c}\right)\right\}=\frac{1}{c} e^{-\delta\left(\frac{t-u}{c}\right)}\left\{k\left(\frac{t-u}{c}\right)+\delta \bar{K}\left(\frac{t-u}{c}\right)\right\},
$$

it follows using (2.4) that (2.6) may be differentiated to give

$$
m_{e}^{\prime}(u)=\frac{\delta}{c} m_{e}(u)+\frac{m(u)-\int_{0}^{u} m(u-y) d H(y)}{c E(V)}-\tau(u),
$$

where, since $c E(V)=(1+\theta) E(Y)$,

$$
\tau(u)=\frac{1}{(1+\theta) E(Y)} \int_{u}^{\infty} w(u, y-u) d H(y) .
$$

We remark that $\tau(u)$ does not depend on $K(t)$ and is thus the same function for any choice of $K(t)$. This invariance property is relevant for what follows in Section 4.

Also, (2.7) is a straightforward first order linear differential equation in $m_{e}(u)$. We shall solve it by taking Laplace transforms in order to take advantage of the convolution terms. Thus, let $\tilde{m}_{e}(s)=\int_{0}^{\infty} e^{-s u} m_{e}(u) d u$, $\tilde{m}(s)=\int_{0}^{\infty} e^{-s u} m(u) d u$, and $\tilde{\tau}(s)=\int_{0}^{\infty} e^{-s u} \tau(u) d u$.

Then from (2.7), it follows that

$$
s \tilde{m}_{e}(s)-m_{e}(0)=\frac{\delta}{c} \tilde{m}_{e}(s)+\frac{\tilde{m}(s)-\tilde{m}(s) \tilde{h}(s)}{c E(V)}-\tilde{\tau}(s) .
$$

Equation (2.9) may be used to derive the Gerber-Shiu defective renewal equation in the classical Poisson model, as is discussed in Section 4.

The following theorem, expressing $m_{e}(u)$ in terms of $m(u)$, is a generalization of (1.1), but retains the same basic structure.

Theorem 2.1 The Gerber-Shiu discounted penalty function $m_{e}(u)$ in the stationary renewal risk model may be expressed in terms of that of the (ordinary) renewal risk model $m(u)$ as

$$
m_{e}(u)=\frac{1}{1+\theta} \int_{0}^{u} m(u-t) d H_{1}(t)+q(u),
$$


where

$$
q(u)=e^{\frac{\delta}{c} u} \int_{u}^{\infty} e^{-\frac{\delta}{c} t}\left\{\tau(t)-\frac{\delta}{c(1+\theta)} \int_{0}^{t} m(t-y) d H_{1}(y)\right\} d t .
$$

Proof: For notational convenience, we introduce the function

$$
\sigma(u)=\int_{0}^{u} m(u-t) d H_{1}(t)
$$

and its Laplace transform

$$
\tilde{\sigma}(s)=\int_{0}^{\infty} e^{-s u} \sigma(u) d u=\tilde{m}(s) \tilde{h}_{1}(s)=\tilde{m}(s) \frac{1-\tilde{h}(s)}{s E(Y)} .
$$

Then, since $E(Y) /\{c E(V)\}=1 /(1+\theta),(2.9)$ may be expressed as

$$
\left(s-\frac{\delta}{c}\right) \tilde{m}_{e}(s)=m_{e}(0)+\frac{1}{1+\theta} s \tilde{\sigma}(s)-\tilde{\tau}(s) .
$$

Put $s=\delta / c$ in (2.14) to obtain

$$
m_{e}(0)=\tilde{\tau}\left(\frac{\delta}{c}\right)-\frac{\delta}{c(1+\theta)} \tilde{\sigma}\left(\frac{\delta}{c}\right)
$$

Substitution of (2.15) into (2.14) results in

In other words,

$$
\begin{aligned}
\left(s-\frac{\delta}{c}\right) \tilde{m}_{e}(s) & =\frac{1}{1+\theta} s \tilde{\sigma}(s)+\tilde{\tau}\left(\frac{\delta}{c}\right)-\tilde{\tau}(s)-\frac{\delta}{c(1+\theta)} \tilde{\sigma}\left(\frac{\delta}{c}\right) \\
& =\frac{\left(s-\frac{\delta}{c}\right)}{1+\theta} \tilde{\sigma}(s)+\tilde{\tau}\left(\frac{\delta}{c}\right)-\tilde{\tau}(s)-\frac{\delta\left\{\tilde{\sigma}\left(\frac{\delta}{c}\right)-\tilde{\sigma}(s)\right\}}{c(1+\theta)} .
\end{aligned}
$$

$$
\tilde{m}_{e}(s)=\frac{1}{1+\theta} \tilde{\sigma}(s)+\frac{\tilde{\tau}\left(\frac{\delta}{c}\right)-\tilde{\tau}(s)}{s-\frac{\delta}{c}}-\frac{\delta}{c(1+\theta)} \frac{\tilde{\sigma}\left(\frac{\delta}{c}\right)-\tilde{\sigma}(s)}{s-\frac{\delta}{c}} .
$$

By Proposition 2.1 with $r=\delta / c,(2.15)$ may be inverted to give

$$
m_{e}(u)=\frac{1}{1+\theta} \sigma(u)+e^{\frac{\delta}{c} u} \int_{u}^{\infty} e^{-\frac{\delta}{c} t} \tau(t) d t-\frac{\delta}{c(1+\theta)} e^{\frac{\delta}{c} u} \int_{u}^{\infty} e^{-\frac{\delta}{c} t} \sigma(t) d t,
$$

which is $(2.10)$.

The general expression (2.10) is somewhat complicated, but is nevertheless useful because its many special cases all have the same basic structure. This will become apparent in the next section. 


\section{The discount free case}

Considerable simplification occurs in the results of the previous section if $\delta=0$. Thus, define

$$
\beta(u)=E\left\{w\left(U_{T-},\left|U_{T}\right|\right) I(T<\infty)\right\}
$$

and

$$
\beta_{e}(u)=E\left\{w\left(U_{T_{e}-},\left|U_{T_{e}}\right|\right) I\left(T_{e}<\infty\right)\right\},
$$

for the ordinary and stationary renewal risk processes respectively. We have the following corollary.

Corollary 3.1 The functions $\beta_{e}(u)$ and $\beta(u)$ defined by (3.2) and (3.1) respectively, are related by

$\beta_{e}(u)=\frac{1}{1+\theta} \int_{0}^{u} \beta(u-t) d H_{1}(t)+\frac{1}{(1+\theta) E(Y)} \int_{u}^{\infty} \int_{t}^{\infty} w(t, y-t) d H(y) d t$

Proof: The result follows from Theorem 2.1 with $\delta=0$, since in this case $q(u)$ in (2.11) becomes $\int_{u}^{\infty} \tau(t) d t$, with $\tau$ given by (2.8).

The case with $\delta=0$ is simpler because the term on the right side of (3.3) does not depend on $K(t)$ or $\beta(u)$. Moreover, the result is sufficiently general to accommodate arbitrary functions of the surplus prior to ruin and the deficit at ruin. It is easy to see that (3.3) reduces to (1.1) when $w\left(x_{1}, x_{2}\right)=1$.

Define

$$
F(u, x, z)=\operatorname{Pr}\left\{T<\infty, U_{T-} \leq x,\left|U_{T}\right| \leq z\right\}
$$

and

$$
F_{e}(u, x, z)=\operatorname{Pr}\left\{T_{e}<\infty, U_{T_{e}-} \leq x,\left|U_{T_{e}}\right| \leq z\right\},
$$

to be the defective joint distribution functions of the surplus prior to ruin and the deficit at ruin in the ordinary and stationary renewal risk models respectively. Their relationship is given in the following corollary.

Corollary 3.2 The functions $F_{e}(u, x, z)$ and $F(u, x, z)$ defined by (3.5) and (3.4) respectively are related by

$$
\begin{aligned}
F_{e}(u, x, z) & =\frac{1}{1+\theta} \int_{0}^{u} F(u-t, x, z) d H_{1}(t) \\
& +\frac{I(u<x)}{1+\theta}\left\{\bar{H}_{1}(u)-\bar{H}_{1}(x)-\bar{H}_{1}(u+z)+\bar{H}_{1}(x+z)\right\}
\end{aligned}
$$


Proof: If $w\left(x_{1}, x_{2}\right)=w_{1}\left(x_{1}\right) w_{2}\left(x_{2}\right)$, then

$$
\int_{u}^{\infty} \int_{t}^{\infty} w(t, y-t) d H(y) d t=\int_{u}^{\infty} w_{1}(t) \int_{t}^{\infty} w_{2}(y-t) d H(y) d t .
$$

Clearly, $F(u, x, z)=E\left\{I(T<\infty) I\left(U_{T-} \leq x\right) I\left(\left|U_{T}\right| \leq z\right)\right\}$, and similarly for $F_{e}(u, x, z)$. Thus choose $w_{1}\left(x_{1}\right)=I\left(x_{1} \leq x\right)$ and $w_{2}\left(x_{2}\right)=I\left(x_{2} \leq z\right)$. Therefore, $w_{2}(y-t)=I(y \leq t+z)$, and it follows that for $u<x$

$$
\begin{aligned}
& \frac{1}{(1+\theta) E(Y)} \int_{u}^{\infty} \int_{t}^{\infty} w(t, y-t) d H(y) d t \\
= & \frac{1}{(1+\theta) E(Y)} \int_{u}^{\infty} w_{1}(t) \int_{t}^{\infty} w_{2}(y-t) d H(y) d t \\
= & \frac{1}{(1+\theta) E(Y)} \int_{u}^{x} \int_{t}^{t+z} d H(y) d t \\
= & \frac{1}{(1+\theta) E(Y)} \int_{u}^{x}\{\bar{H}(t)-\bar{H}(t+z)\} d t \\
= & \frac{1}{1+\theta}\left\{\bar{H}_{1}(u)-\bar{H}_{1}(x)-\bar{H}_{1}(u+z)+\bar{H}_{1}(x+z)\right\} .
\end{aligned}
$$

The integral is clearly 0 if $u \geq x$.

The defective marginal distribution function of the surplus prior to ruin is obtained with $z=\infty$, and for the deficit at ruin with $x=\infty$. The latter distribution is implicit in Willmot et al (2002).

Moments of the deficit at ruin are easily obtained with the choice $w_{1}(x)=$ 1 and $w_{2}(x)=x^{k}$. In this case the right hand term in (3.3) may be expressed in terms of higher order equilibrium distributions of the $\mathrm{df} H(y)$ as in Lin and Willmot (2000). The details are omitted. For a different approach, see Willmot et al (2002).

In the next section we consider the classical model and its relationship to the stationary model.

\section{The classical Poisson model revisited}

In the special case with $K(t)=1-e^{-\lambda t}$, one has $E(V)=1 / \lambda$, and $m_{e}(u)=$ $m(u)=m_{*}(u)$, where $m_{*}(u)$ is given by (1.3). Let $\tilde{m}_{*}(s)=\int_{0}^{\infty} e^{-s u} m_{*}(u) d u$, and (2.9) becomes

$$
\left\{s+\frac{\lambda}{c} \tilde{h}(s)-\frac{\lambda+\delta}{c}\right\} \tilde{m}_{*}(s)=m_{*}(0)-\tilde{\tau}(s) .
$$


Now, from (1.4) the left side of (4.1) vanishes when $s=\rho$. Therefore

$$
m_{*}(0)=\tilde{\tau}(\rho) .
$$

Also, the Laplace transform of the $\mathrm{df} B(x)$ defined by (1.6) is (e.g. Willmot and Lin, 2001, p.164)

$$
\tilde{b}(s)=\frac{\rho}{1-\tilde{h}(\rho)} \frac{\tilde{h}(\rho)-\tilde{h}(s)}{s-\rho} .
$$

Therefore, again using (1.4)

$$
\begin{aligned}
& s+\frac{\lambda}{c} \tilde{h}(s)-\frac{\lambda+\delta}{c} \\
= & \frac{c s+\lambda \tilde{h}(s)-c \rho-\lambda \tilde{h}(\rho)}{c} \\
= & (s-\rho)\left\{1-\frac{\lambda}{c} \frac{\tilde{h}(\rho)-\tilde{h}(s)}{s-\rho}\right\} \\
= & (s-\rho)\left\{1-\frac{\lambda}{c} \frac{1-\tilde{h}(\rho)}{\rho} \tilde{b}(s)\right\} .
\end{aligned}
$$

But $c=\lambda E(Y)(1+\theta)$, and from (1.5) one has

$$
s+\frac{\lambda}{c} \tilde{h}(s)-\frac{\lambda+\delta}{c}=(s-\rho)\{1-\phi \tilde{b}(s)\} .
$$

Therefore, using (4.2) and (4.4), (4.1) may be expressed as

$$
(s-\rho)\{1-\phi \tilde{b}(s)\} \tilde{m}_{*}(s)=\tilde{\tau}(\rho)-\tilde{\tau}(s),
$$

i.e.

$$
\tilde{m}_{*}(s)=\phi \tilde{m}_{*}(s) \tilde{b}(s)+\frac{\tilde{\tau}(\rho)-\tilde{\tau}(s)}{s-\rho} .
$$

Inversion of (4.5) using Proposition 2.1 yields

$$
m_{*}(u)=\phi \int_{0}^{u} m_{*}(u-t) d B(t)+e^{\rho u} \int_{u}^{\infty} e^{-\rho t} \tau(t) d t .
$$

Clearly, from (2.8) and (1.8), it follows that (4.6) is the Gerber-Shiu defective renewal equation (1.7).

Next, define

$$
\beta_{*}(u)=E\left\{w\left(U_{T_{*}-},\left|U_{T_{*}}\right|\right) I\left(T_{*}<\infty\right)\right\}
$$


Thus $\beta_{*}(u)=m_{*}(u)$ when $\delta=0$, and $\beta_{*}(u)=\beta_{e}(u)=\beta(u)$ when $K(t)=$ $1-e^{-\lambda t}$. Therefore, (3.3) is a defective renewal equation for $\beta_{*}(u)$ when $K(t)=1-e^{-\lambda t}$. Similarly, when $\delta=0, \rho=0, \phi=1 /(1+\theta)$, and $B(x)=$ $H_{1}(x)$ from (1.6). This implies that (1.7), (4.6), and (3.3) are all the same defective renewal equation when $\delta=0$ and $K(t)=1-e^{-\lambda t}$. However, when $\delta>0,(2.10)$ differs from (1.7) and (4.6) when $K(t)=1-e^{-\lambda t}$, since in this case $q(u)$ in (2.11) depends on $m_{*}(u)$.

The following corollary gives an invariance property which generalizes (1.2).

Corollary 4.1 Let $\beta_{e}(u)$ defined by (3.2) and $\beta_{*}(u)$ defined by (4.7) be the Gerber-Shiu penalty function with no discount factor in the stationary renewal risk models and classical Poisson models respectively. Then

$$
\beta_{e}(0)=\beta_{*}(0)=\frac{1}{(1+\theta) E(Y)} \int_{0}^{\infty} \int_{t}^{\infty} w(t, y-t) d H(y) d t
$$

Proof: The outer equality in (4.8) follows from (3.3) with $u=0$. Also, when $\delta=0, \rho=0$, and thus when $\delta=0,(4.2)$ implies that $\beta_{*}(0)=m_{*}(0)=$ $\tilde{\tau}(0)=\int_{0}^{\infty} \tau(t) d t$, and the result follows from (2.8).

We remark that (4.8) does not depend on $K(t)$. Also, (1.2) is recovered when $w(\cdot)=1$. Furthermore, $m_{e}(0)$ is given by $(2.15)$ and $m_{*}(0)$ by (4.2), so that $m_{e}(0) \neq m_{*}(0)$ unless $\delta=0$, in which case both equal $\tilde{\tau}(0)$. Thus, (4.8) may not be extended to the discounted case.

Also, let the defective joint distribution function of the surplus prior to ruin and the deficit at ruin in the classical Poisson model be

$$
F_{*}(u, x, z)=\operatorname{Pr}\left\{T_{*}<\infty, U_{T_{*}-} \leq x,\left|U_{T_{*}}\right| \leq z\right\} .
$$

Then from (3.6) with $u=0$ and (4.8), it follows that (3.5) and (4.9) are related for $x \geq 0$ and $z \geq 0$ by

$$
F_{e}(0, x, z)=F_{*}(0, x, z)=\frac{1-\bar{H}_{1}(x)-\bar{H}_{1}(z)+\bar{H}_{1}(x+z)}{1+\theta} .
$$

As in the case with (3.6), corresponding relationships with $u=0$ for the defective marginal distribution functions of the surplus prior to ruin and the deficit at ruin follows from (4.10) with $z=\infty$ and $x=\infty$ respectively.

\section{References}

Asmussen, S. (2000). Ruin Probabilities. World Scientific, Singapore. 
Dickson, D., and C. Hipp (2001). "On the time to ruin for Erlang (2) risk processes." Insurance: Mathematics and Economics 29, 333-344.

Drekic, S., D. Dickson, D. Stanford, and G. Willmot (2002). "On the distribution of the deficit at ruin when claims are phase-type." Scandinavian Actuarial Journal, to appear.

Embrechts, P., C. Klüppelberg, and T. Mikosch (1997). Modelling Extremal Events. Springer-Verlag, Berlin.

Gerber, H., and E. Shiu (1998). "On the time value of ruin." North American Actuarial Journal 2, 48-78.

Grandell, J. (1991). Aspects of Risk Theory. Springer-Verlag, New York.

Lin, X. and G. Willmot (1999). "Analysis of a defective renewal equation arising in ruin theory." Insurance: Mathematics and Economics 25, $63-84$.

Lin, X. and G. Willmot (2000). "The moments of the time of ruin, the surplus before ruin, and the deficit at ruin." Insurance: Mathematics and Economics 27, 19-44.

Rolski, T., H. Schmidli, V. Schmidt, and J. Teugels (1999). Stochastic Processes for Insurance and Finance. John Wiley, Chichester.

Willmot, G. and X. Lin (2001). Lundberg Approximations for Compound Distributions with Insurance Applications. Springer-Verlag, New York.

Willmot, G., D. Dickson, S. Drekic, and D. Stanford (2002). "The deficit at ruin in the stationary renewal risk model." Institute of Insurance and Pension Research Report 02-10, University of Waterloo, Waterloo, Ontario, Canada. 
RECENT ADDITIONS TO THE RESEARCH PAPER SERIES

No.

Date

FEBRUARY 2001

FEBRUARY 2001

JUNE 2001

87
SEPTEMBER 2001

NOVEMBER 2001

NOVEMBER 2001

NOVEMBER 2001

JANUARY 2002

JANUARY 2002

JANUARY 2002

JANUARY 2002

MAY 2002

JUNE 2002

JUNE 2002
Subject

Author

DISCRETE TIME RISK MODELS UNDER STOCHASTIC FORCES OF INTEREST

MODERN LANDMARKS IN ACTUARIAL SCIENCE Inaugural Professorial Address

LUNDBERG INEQUALITIES FOR RENEWAL EQUATIONS

VOLATILITY, BETA AND RETURN WAS THERE EVER A MEANINGFUL RELATIONSHIP?

EXPLICIT, FINITE TIME RUIN PROBABILITIES FOR DISCRETE, DEPENDENT CLAIMS

ON THE DISTRIBUTION OF THE DEFICIT AT RUIN WHEN CLAIMS ARE PHASE-TYPE

THE INTEGRATED SQUARE-ROOT PROCESS

ON THE EXPECTED DISCOUNTED PENALTY FUNCTION AT RUIN OF A SURPLUS PROCESS WITH INTEREST

\section{CHAIN LADDER BIAS}

FURTHER OBSERVATIONS ON CHAIN LADDER BIAS

A GENERAL CLASS OF RISK MODELS

THE DISTRIBUTION OF THE TIME TO RUIN IN THE CLASSICAL RISK MODEL

A NOTE ON THE MAXIMUM SEVERITY OF RUIN AND RELATED PROBLEMS

UPPER BOUNDS FOR ULTIMATE RUIN PROBABILITIES IN THE SPARRE ANDERSEN MODEL WITH INTEREST

CONTINUOUS COMPOUNDING, VOLATILITY AND THE EQUITY PREMIUM
Jun Cai

David C M Dickson

Gordon E Willmot Jun Cai

$\mathrm{X}$ Sheldon Lin

Richard Fitzherbert

Zvetan G Ignatov Vladimir K Kaishev Rossen S Krachunov

Steve Drekic

David C M Dickson David A Stanford Gordon E Willmot

Daniel Dufresne

Jun Cai

David C M Dickson

Greg Taylor

Greg Taylor

Daniel Dufresne

David C M Dickson Howard R Waters

David C M Dickson Jun Cai

David C M Dickson

Richard Fitzherbert 
No.

Date

99

JUNE 2002

THE DEFICIT AT RUIN IN THE STATIONARY RENEWAL RISK MODEL

$\begin{array}{ll}100 & \text { AUGUST 2002 } \\ 101 & \text { AUGUST 2002 } \\ 102 & \text { AUGUST 2002 }\end{array}$

Subject

Author

Gordon E Willmot David C M Dickson Steve Drekic

David A Stanford

Daniel Dufresne

ASIAN AND BASKET ASYMPTOTICS

RUIN PROBABILITIES WITH A MARKOV CHAIN INTEREST MODEL

Jun Cai

David C M Dickson

Gordon E Willmot

David C M Dickson FUNCTION IN THE STATIONARY RENEWAL RISK MODEL

A complete list of papers can be found at http://www.economics.unimelb.edu.au/actwww/papers.html 


\section{University Library}

\section{- M M I N E R VA A gateway to Melbourne's research publications}

Minerva Access is the Institutional Repository of The University of Melbourne

Author/s:

Willmot, Gordon E.;Dickson, David C. M.

Title:

The Gerber-Shiu discounted penalty function in the stationary renewal risk model

Date:

2002-08

Citation:

Willmot, Gordon E. and Dickson, David C. M. (2002) The Gerber-Shiu discounted penalty function in the stationary renewal risk model.

Persistent Link:

http://hdl.handle.net/11343/33694 